\title{
Involvement of the optic nerve in mutated CSF1R-induced hereditary diffuse leukoencephalopathy with axonal spheroids
}

Yaqing Shu ${ }^{1 \dagger}$, Ling Long ${ }^{1 \dagger}$, Siyuan Liao ${ }^{1}$, Jiezheng Yang ${ }^{2}$, Jianfang $\mathrm{Li}^{3}$, Wei Qiu' ${ }^{1}$, Yu Yang ${ }^{1}$, Jian Bao ${ }^{1}$, Aiming Wu${ }^{1}$, Xuegiang $\mathrm{Hu}^{1 *}$ and Zhengqi Lü

\begin{abstract}
Background: Hereditary diffuse leukoencephalopathy with axonal spheroids (HDLS) is a rare autosomal dominant disorder characterized by cerebral white matter degeneration and caused by mutations in the colony-stimulating factor 1 receptor (CSF1R) gene. Involvement of the optic nerves in hereditary diffuse leukoencephalopathy is rare.

Case presentation: We report the case of a 30-year-old Chinese woman with HDLS, who carried a heterozygous c.2345 G > A (p.782Arg > His) mutation in exon 18 of CSF1R. She developed a gradual decline in motor ability, as well as cognitive and visual function, over the course of 4 months. Brain T2 fluid-attenuated inversion recovery-weighted magnetic resonance imaging revealed high signal lesions in the bilateral frontoparietal and periventricular deep white matter. Optical coherence tomography showed that the right peripapillary retinal nerve fiber layer was atrophic in the temporal quadrant while the left peripapillary retinal nerve fiber layer was thin in the temporal superior quadrant.
\end{abstract}

Conclusions: A diagnosis of HDLS should be considered in patients with white matter lesions and optic nerves injury upon magnetic resonance imaging that mimics progressive multiple sclerosis.

Keywords: Leukoencephalopathy, Colony-stimulating factor 1 receptor, Peripapillary retinal nerve fiber layer, Hereditary diffuse leukoencephalopathy with axonal spheroids

\section{Background}

Hereditary diffuse leukoencephalopathy with axonal spheroids (HDLS) is a rare autosomal dominant disorder characterized by cerebral white matter degeneration with axonal spheroids with variable clinical presentations, including personality and behavioral changes, dementia, depression, parkinsonism, and seizures. HDLS was first identified in a Western Swedish family reported in 1984 [1] and is caused by mutations in the colony-stimulating factor 1 receptor (CSF1R) gene [2]. HDLS appears as white matter lesions in a frontal predominant distribution upon brain magnetic resonance imaging (MRI), spreading out from the periventricular and deep white

\footnotetext{
* Correspondence: huxueqiangqm@aliyun.com; Izq1828@aliyun.com ${ }^{\dagger}$ Equal contributors

1 Department of Neurology, The Third Affiliated Hospital of Sun Yat-sen University, 600 Tianhe Road, Guangzhou 510630, China

Full list of author information is available at the end of the article
}

matter into the subcortical areas with enlarged ventricles and often signal changes in the corpus callosum [3, 4]. However, involvement of the optic nerves has not been previously reported in HDLS. We describe a HDLS patient who also had optic nerve lesions.

\section{Case presentation}

A 30-year-old, non-smoking woman was referred to our hospital in August 2015, with a 4-month history of progressive lower limb weakness, gait disturbance, and visual disorder. Her mother experienced right lower limb weakness at 40 years of age, followed by progressive cognitive and motor deterioration and death at 60 years of age. The case family pedigree is shown in Fig. 1.

Prior to admission to our hospital, the patient exhibited neuropsychiatric symptoms, such as paraphasia and hallucination. She was diagnosed with multiple sclerosis 


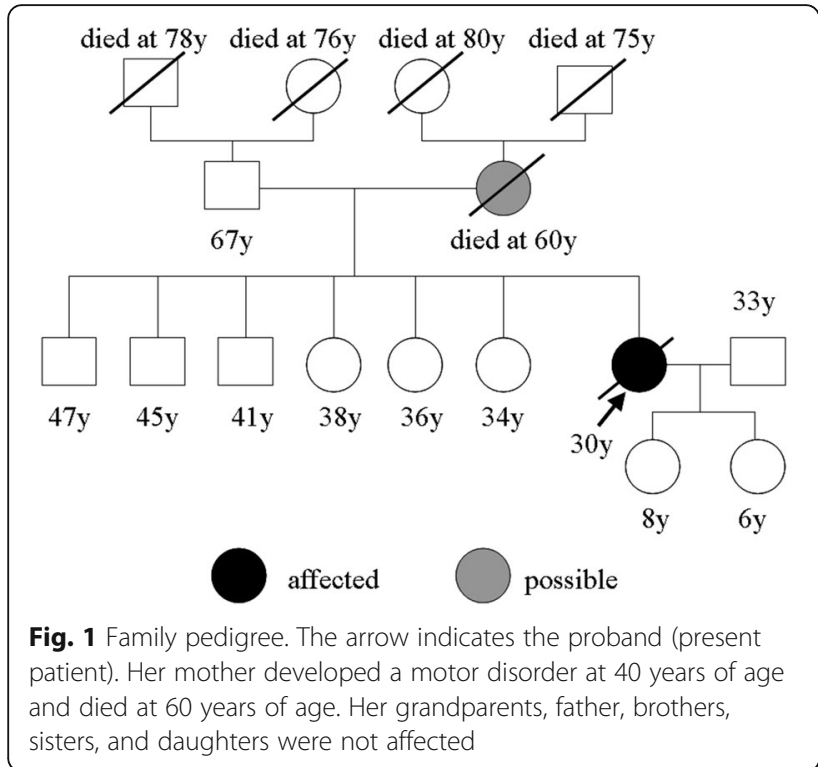

(MS) and received immunotherapy with intravenous methylprednisolone at another hospital. However, her motor function progressively declined and she experienced frequent falls. When she was admitted to our hospital, her mini-mental state examination (MMSE) score was 25/30, with a Montreal cognitive assessment scale (MoCA) score of $17 / 30$. The activities of daily living (ADL) score was 50/100 and the Morse fall risk factors assessment scale score was 45 . Bilateral visual acuity was
0.8, muscle tone in her lower limbs increased, and muscle strength of the lower limbs was grade 4. She developed a spastic gait and an unsteady broad-based gait. Romberg's sign, Babinski's sign, and Chaddock's sign were all positive.

Most laboratory tests were normal/negative, including the serum erythrocyte sedimentation rate, $\mathrm{C}$-reactive pro-

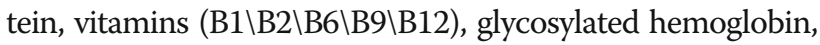
thyroid function, serum copper, ceruloplasmin, antinuclear antibody, paraneoplastic antibodies, and antibodies specific for the following: myelin oligodendrocyte glycoprotein, myelin-associated glycoprotein, myelin basic protein, and aquaporin-4. Serum treponema pallidum antibody, rapid plasma reagin, tolulized red unheated serum test, and human immunodeficiency virus antibody were all negative. Cerebrospinal fluid tests were normal, including negative oligoclonal IgG bands.

Brain T2 fluid-attenuated inversion recovery (FLAIR) MRI showed multiple, asymmetrical, hyperintense lesions, which appeared in periventricular areas and the white matter regions of the frontal and parietal lobes. Diffusionweighted imaging (DWI) showed small dots of diffusion restriction within the white matter lesions. Magnetic resonance spectroscopy (MRS) showed increased levels of choline (Cho), although $\mathrm{N}$-acetylaspartate (NAA) was decreased. Diffusion tensor imaging (DTI) revealed corpus callosum fiber loss, although fibers of the bilateral frontal, parietal, occipital, and temporal lobe and brainstem fibers were spared (Fig. 2).

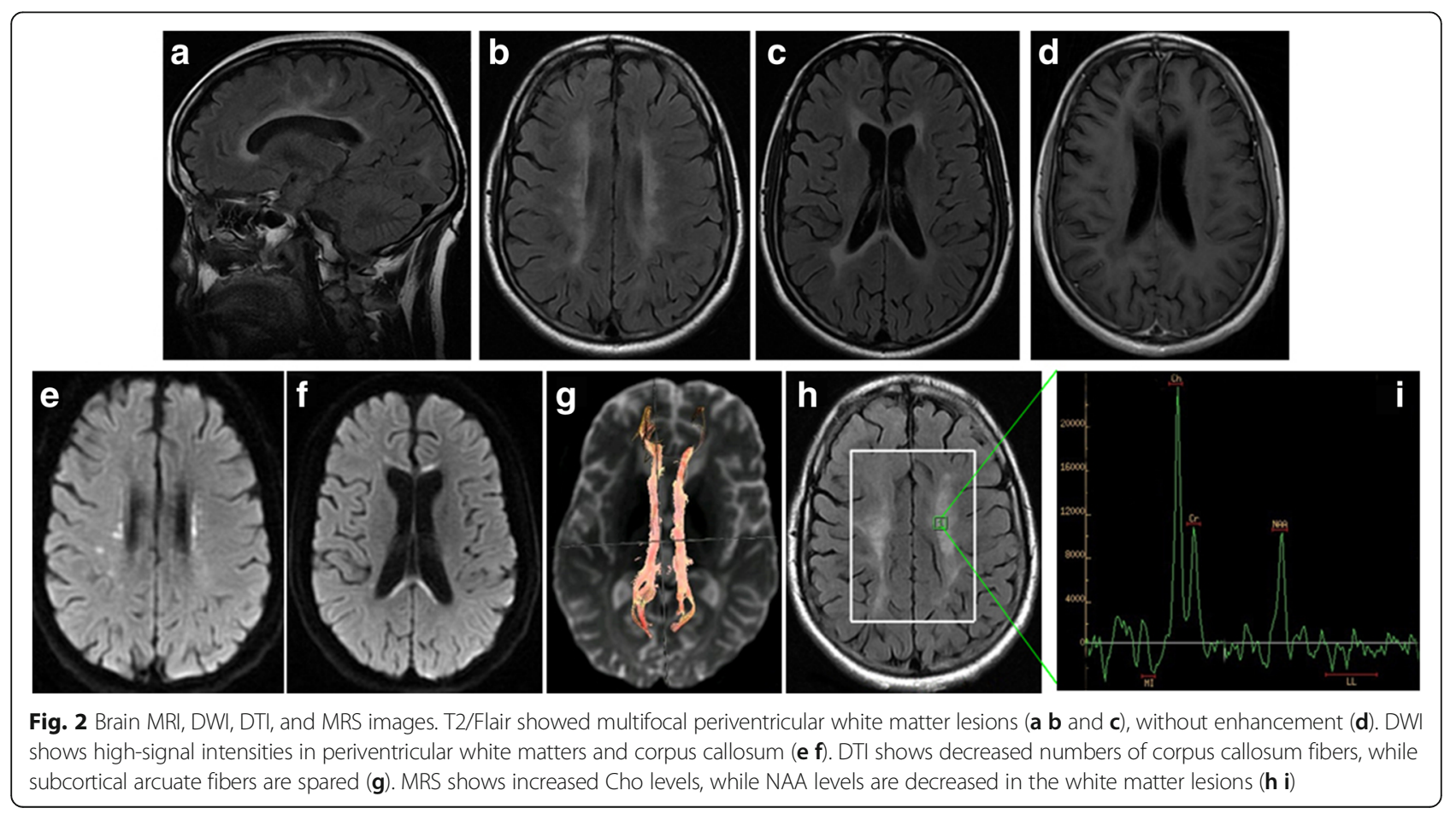




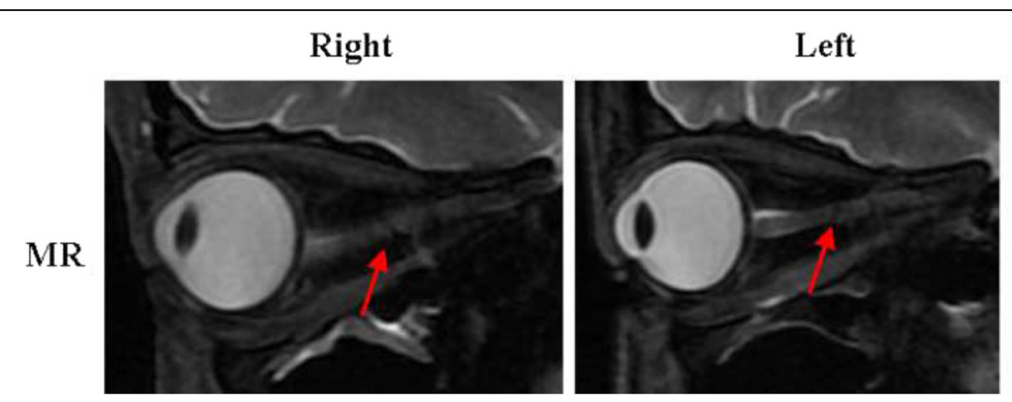

Fig. 3 Optic nerves on MRI, showing that bilateral optic nerves are injured (red arrows)

Optic nerve MRI demonstrated bilateral optic nerves lesions (Fig. 3). Optical coherence tomography (OCT) showed that the right peripapillary retinal nerve fiber layer (pRNFL) was atrophic (pRNFL thickness $43-50 \mu \mathrm{m}$ ) in the temporal quadrant, and the left pRNFL was thin $(65 \mu \mathrm{m})$ in the temporal superior quadrant (Fig. 4). Average RNFL thickness in healthy controls has been shown to be 93-108 $\mu \mathrm{m}$ [5]. Visual-evoked potential (VEP) showed reduced amplitudes of bilateral P100, although latencies of bilateral P100 were normal (Fig. 5). Bilateral intraocular pressures (both $15 \mathrm{mmHg}$ ) were normal. The visual fields of both eyes were partially missing in the different quadrants (Fig. 6).
Methylprednisolone therapy with two treatment courses $(1000 \mathrm{mg} /$ day for 5 days and $1000 \mathrm{mg} /$ day for 3 days) resulted in no improvement. Genetic studies for leukodystrophy-related genes showed that a c.2345 G > A (p.782Arg > His) mutation in exon 18 of CSF1R was present (Fig. 7), which has been proven to be the pathogenic gene for HDLS. However, a brain biopsy was refused by the patient and her family. Seven months after discharge from our hospital, she showed progressive decline and reached a vegetative state and died on May 18, 2016.

The present case describes a patient with a genetically confirmed HDLS and a heterozygous c. 2345 G > A

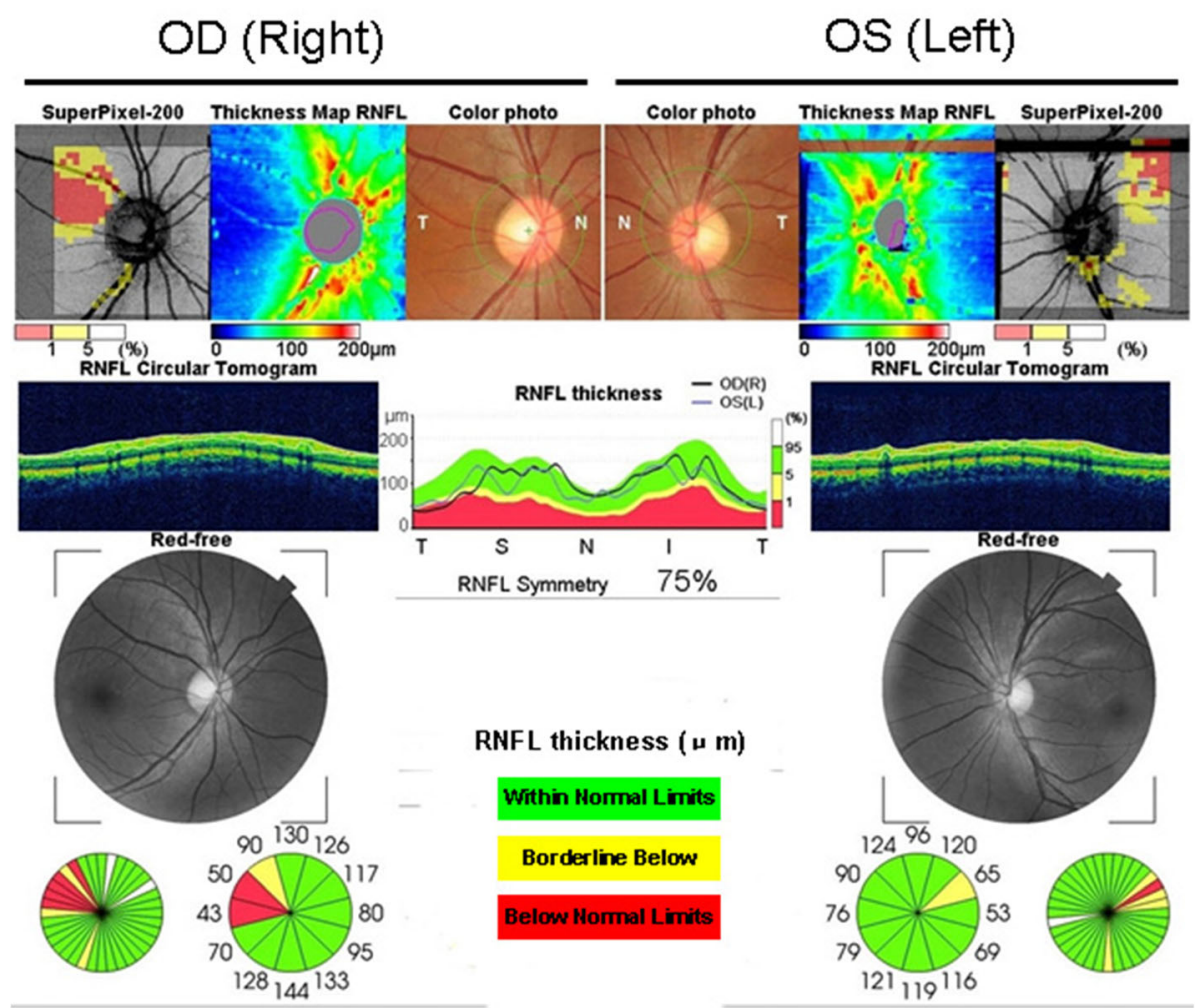

Fig. 4 OCT shows that the right peripapillary retinal nerve fiber layer ( $p R N F L$ ) is atrophic in the temporal quadrant, and the left pRNFL is thinning in the temporal superior quadrants. Green represents pRNFL thickness, which is within normal limits; yellow represents pRNFL thickness, which is below borderline; red represents PRNFL thickness, which is below normal limits 


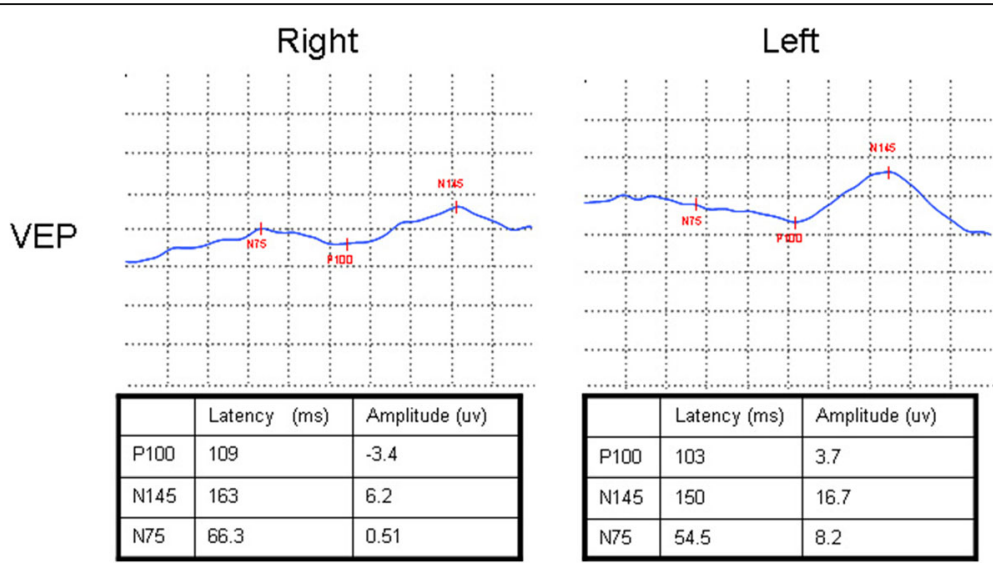

Fig. 5 VEP shows reduced bilateral P100 amplitudes, although P100 latencies are normal in both eyes

(p.782Arg > His) mutation in exon 18 of the CSFR1, which was previously described by Kinoshita et al. [6]. HDLS has an autosomal dominant pattern of inheritance, and it may be sporadic. In our case, we presume that the patient's mother was affected, because of early-onset motor impairment over the course of her illness. Unfortunately, however, there were no clinical data or brain biopsy samples from her mother.
In the present case, the patient experienced visual impairment and optic nerve impairment, which made it difficult to differentiate the disease from MS. Optic nerve injury lacking gadolinium enhancement was revealed by brain MRI, and the right temporal pRNFL was atrophic and left temporal superior pRNFL was thin upon OCT. To the best of our knowledge, involvement of the optic nerves has never been reported in HDLS,

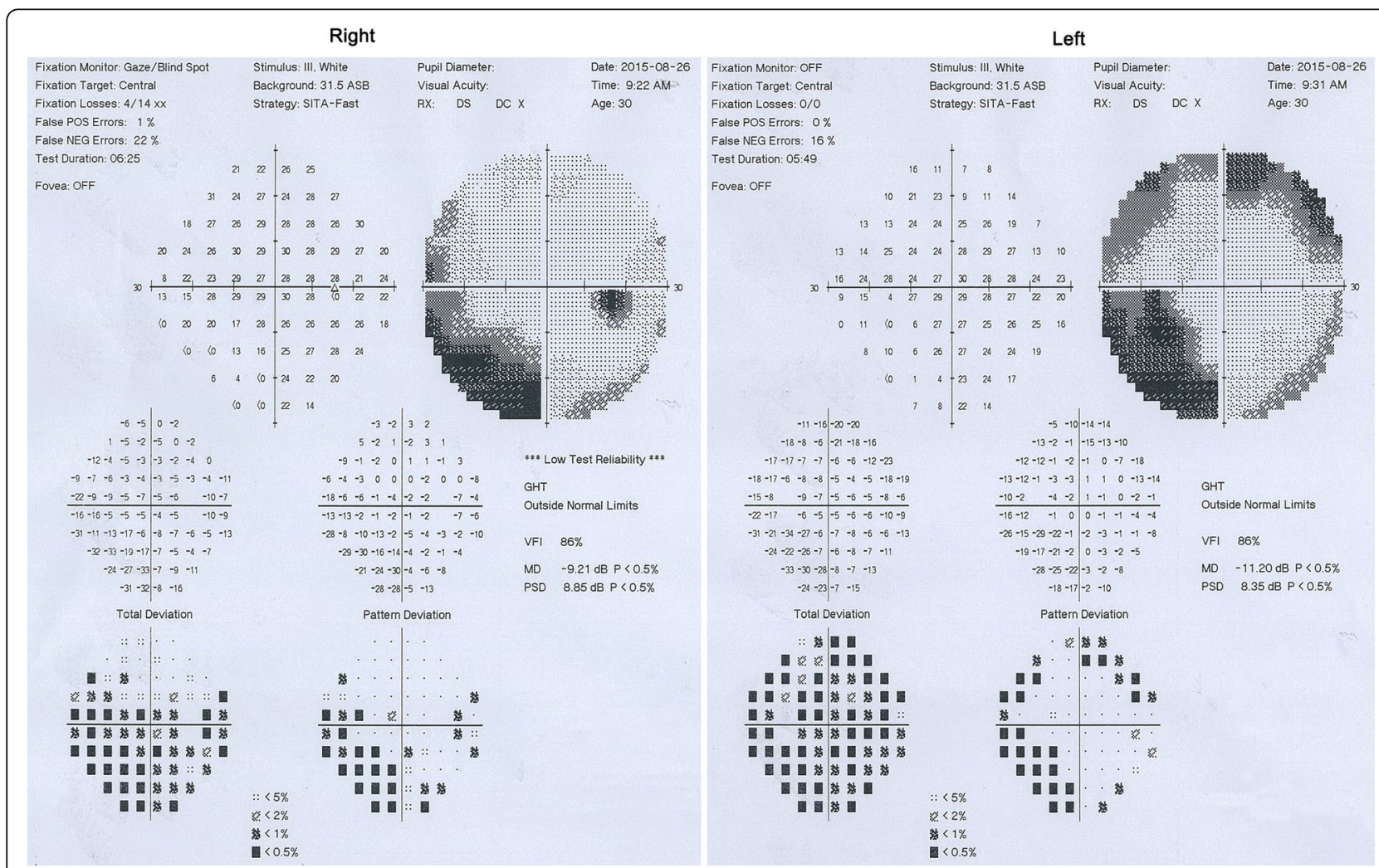

Fig. 6 Visual fields in the right eye are partially missing in the upper right, lower right, and lower left quadrants, especially in the lower right quadrant. Visual fields in the left eye are partially missing in the four quadrants, especially in the upper left and lower right quadrants 


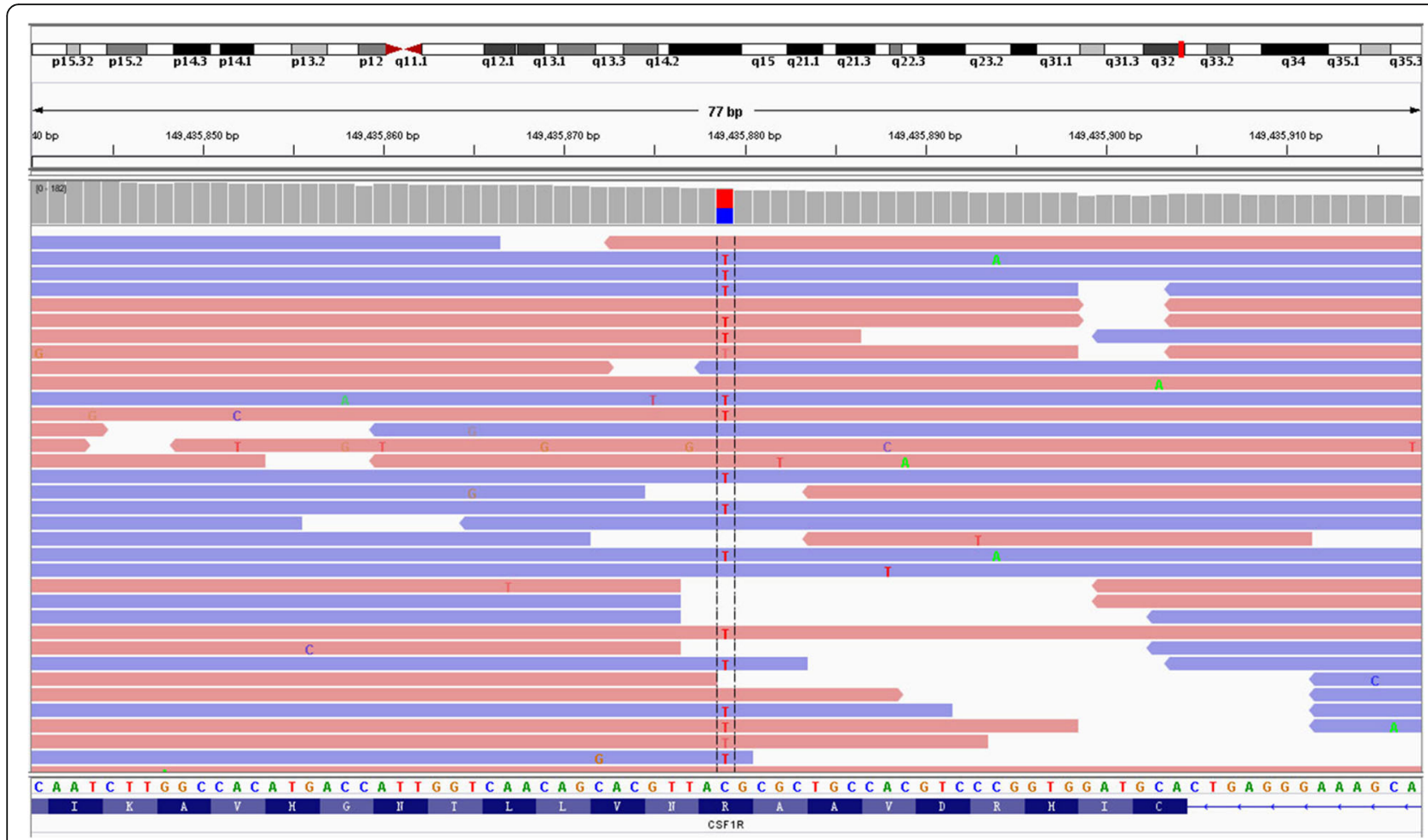

Fig. 7 Gene analysis of CSF1R. The sequencing result from exon 18 of CSF1R (NM_005211.3) indicates a heterozygous c.2345 G > A (p.782Arg > His) substitution in the patient

and the mechanism of optic nerve impairment in HDLS remains unknown. HDLS is pathologically characterized by non-inflammatory myelin loss, reactive astrocytosis, and axonal spheroids. Axonal spheroids of peripheral neuropathy have been found in HDLS, suggesting more widespread nervous system involvement [7]. We hypothesized that non-inflammatory myelin loss and axonal spheroids might also be involved in the optic nerves in our HDLS case. Additionally, OCT, which has emerged as a novel tool to depict neuro-axonal damage in MS by measuring non-myelinated central nervous system axons in the retina $[5,8]$, was also used to analyze RNFL thickness that is the better parameter for monitoring longitudinal axonal damage [9]. RNFL thickness has also been linked to metabolic signs of neurodegeneration in the visual cortex [10]. Several reports of MS patients have correlated RNFL atrophy with brain atrophy in patients with and without optic neuritis $[8,9,11]$. In our case, brain MRI showed multiple lesions in the white and gray matter, and DTI revealed lost or spared white and gray matter fibers. These results suggested that RNFL atrophy in OCT might be associated with brain abnormalities in HDLS. We hypothesized that the features revealed by OCT and neuroimaging might be related to axonal and myelin destruction in white matter lesions in HDLS.

The clinical features in the present case predominantly included progressive gait disturbance and cognitive and motor impairment that mimicked progressive MS, although the patient also exhibited atypical parkinsonian features, including bradykinesia and rigidity, which have been previously reported [12-14]. Although the mechanisms of atypical Parkinsonism remain unclear, it has been suggested that the disconnected fiber tracts in white matter lesions are structurally or functionally critical for performing movements $[15,16]$. Subcortical and periventricular white matter lesions might interfere with ascending thalamocortical and descending corticospinal fibers, and a large number of white matter lesions have been observed in HDLS.

\section{Conclusions}

Results from the present case study suggest that optic nerves can be involved in HDLS. RNFL atrophy observed by OCT might be associated with brain abnormalities in HDLS. Molecular analysis of the CSF1R gene should be performed when HDLS clinically and radiologically mimics progressive multiple sclerosis.

\section{Abbreviations}

ADL: Activities of daily living; Choline: Cho; CSF1R: Colony stimulating factor 1 receptor; DTI: Diffusion tensor imaging; DWI: Diffusion weighted imaging; HDLS: Hereditary diffuse leukoencephalopathy with axonal spheroids; MMSE: Mini-mental state examination; MoCA: Montreal cognitive assessment scale; MRI: Magnetic resonance imaging; MRS: Magnetic resonance spectroscopy; MS: Multiple sclerosis; NAA: N-acetylaspartate; OCT: Optical 
coherence tomography; pRNFL: Peripapillary retinal nerve fiber layer; T2 FLAIR: T2 fluid-attenuated inversion recovery

\section{Acknowledgements}

We wish to thank our patient and her family.

\section{Funding}

The study was supported by grants from the National Natural Science Foundation of China (no. 81301088)

\section{Availability of data and materials}

The datasets supporting the conclusions of this article are available in the zenodo repository. Figures are available on https://zenodo.org/record/57426.

\section{Authors' contributions}

$\mathrm{ZQ}-\mathrm{L}$ and $\mathrm{XQ}-\mathrm{H}$ interviewed, diagnosed and treated the patient. YQ-S and LL interpreted the data and drafted the manuscript for content. JZ- Y carried our eyes test in this patient. JF-L did the MRI for the patients. SY-L and WQ interviewed the patient and did the Gene analysis for the patient. YY, JB and AM-W contributed to the follow-up of the patients and literature reviewing. All authors read and approved the final manuscript.

\section{Competing interests}

The authors declare that they have no competing interests.

\section{Consent for publication}

Written informed consent was obtained from the patient for publication of this case report and any accompanying images. A copy of the written consent is available for review by the Editor-in-Chief of this journal.

\section{Ethics approval and consent to participate}

This research was approved by the ethics committee of the Third Affiliated Hospital of Sun Yat-sen University. The patient consented to participate in this study.

\section{Author details}

${ }^{1}$ Department of Neurology, The Third Affiliated Hospital of Sun Yat-sen University, 600 Tianhe Road, Guangzhou 510630, China. 'Department of Ophthalmology, The Third Affiliated Hospital of Sun Yat-sen University, Guangzhou 510630, China. ${ }^{3}$ Department of Nuclear Medicine, The Third Affiliated Hospital of Sun Yat-sen University, Guangzhou 510630, China.

\section{Received: 11 April 2016 Accepted: 1 September 2016}

\section{Published online: 13 September 2016}

\section{References}

1. Axelsson R, Roytta M, Sourander P, Akesson HO, Andersen O. Hereditary diffuse leucoencephalopathy with spheroids. Acta Psychiatr Scand Suppl. 1984;314:1-65.

2. Rademakers R, Baker M, Nicholson AM, Rutherford NJ, Finch N, Soto-Ortolaza A, Lash J, Wider C, Wojtas A, DeJesus-Hernandez M, et al. Mutations in the colony stimulating factor 1 receptor (CSF1R) gene

cause hereditary diffuse leukoencephalopathy with spheroids. Nat Genet 2012;44(2):200-5.

3. Kondo Y, Kinoshita M, Fukushima K, Yoshida K, Ikeda S. Early involvement of the corpus callosum in a patient with hereditary diffuse leukoencephalopathy with spheroids carrying the de novo K793T mutation of CSF1R. Intern Med. 2013:52(4):503-6.

4. Sundal C, Van Gerpen JA, Nicholson AM, Wider C, Shuster EA, Aasly J, Spina S, Ghetti B, Roeber S, Garbern J, et al. MRI characteristics and scoring in HDLS due to CSF1R gene mutations. Neurology. 2012;79(6):566-74.

5. Bennett JL, de Seze J, Lana-Peixoto M, Palace J, Waldman A, Schippling S, Tenembaum S, Banwell B, Greenberg B, Levy M, et al. Neuromyelitis optica and multiple sclerosis: Seeing differences through optical coherence tomography. Mult Scler. 2015;21(6):678-88.

6. Kinoshita M, Yoshida K, Oyanagi K, Hashimoto T, Ikeda S. Hereditary diffuse leukoencephalopathy with axonal spheroids caused by $\mathrm{R} 782 \mathrm{H}$ mutation in CSF1R: case report. J Neurol Sci. 2012;318(1-2):115-8.

7. Di Donato I, Stabile C, Bianchi S, Taglia I, Mignarri A, Salvatore S, Giorgio E, Brusco A, Simone I, Dotti MT, et al. A Novel CSF1R Mutation in a Patient with Clinical and Neuroradiological Features of Hereditary
Diffuse Leukoencephalopathy with Axonal Spheroids. J Alzheimers Dis. 2015:47(2):319-22

8. Zimmermann H, Freing A, Kaufhold F, Gaede G, Bohn E, Bock M, Oberwahrenbrock T, Young KL, Dorr J, Wuerfel JT, et al.

Optic neuritis interferes with optical coherence tomography and magnetic resonance imaging correlations. Mult Scler. 2013;19(4):443-50.

9. Dorr J, Wernecke KD, Bock M, Gaede G, Wuerfel JT, Pfueller CF, Bellmann-Strobl J, Freing A, Brandt AU, Friedemann P. Association of retinal and macular damage with brain atrophy in multiple sclerosis. PLoS One. 2011;6(4):e18132

10. Pfueller CF, Brandt AU, Schubert F, Bock M, Walaszek B, Waiczies H, Schwenteck T, Dorr J, Bellmann-Strobl J, Mohr C. et al. Metabolic changes in the visual cortex are linked to retinal nerve fiber layer thinning in multiple sclerosis. PLoS One. 2011;6(4):e18019.

11. Young KL, Brandt AU, Petzold A, Reitz LY, Lintze F, Paul F, Martin R, Schippling S. Loss of retinal nerve fibre layer axons indicates white but not grey matter damage in early multiple sclerosis. Eur J Neurol. 2013;20(5):803-11.

12. Saitoh BY, Yamasaki R, Hayashi S, Yoshimura S, Tateishi T, Ohyagi Y, Murai H, Iwaki T, Yoshida K, Kira J. A case of hereditary diffuse leukoencephalopathy with axonal spheroids caused by a de novo mutation in CSF1R masquerading as primary progressive multiple sclerosis. Mult Scler. 2013;19(10):1367-70.

13. Keegan BM, Giannini C, Parisi JE, Lucchinetti CF, Boeve BF, Josephs KA. Sporadic adult-onset leukoencephalopathy with neuroaxonal spheroids mimicking cerebral MS. Neurology. 2008;70(13 Pt 2):1128-33.

14. Sundal C, Fujioka S, Van Gerpen JA, Wider C, Nicholson AM, Baker M, Shuster EA, Aasly J, Spina S, Ghetti B, et al. Parkinsonian features in hereditary diffuse leukoencephalopathy with spheroids (HDLS) and CSF1R mutations. Parkinsonism Relat Disord. 2013;19(10):869-77.

15. Bohnen NI, Albin RL. White matter lesions in Parkinson disease. Nat Rev Neurol. 2011;7(4):229-36.

16. Bonilha L, de Vries PM, Hurd MW, Rorden C, Morgan PS, Besenski N, Bergmann KJ, Hinson VK. Disrupted thalamic prefrontal pathways in patients with idiopathic dystonia. Parkinsonism Relat Disord. 2009;15(1):64-7.

\section{Submit your next manuscript to BioMed Central and we will help you at every step:}

- We accept pre-submission inquiries

- Our selector tool helps you to find the most relevant journal

- We provide round the clock customer support

- Convenient online submission

- Thorough peer review

- Inclusion in PubMed and all major indexing services

- Maximum visibility for your research

Submit your manuscript at www.biomedcentral.com/submit 